\title{
AESTHETIC APPROACH FOR EXTRACTED UPPER PRIMARY INCISORS IN A 4 1⁄2 YEAR-OLD CHILD - A CASE REPORT
}

\author{
Dr P.K. Aravind, Dr Vignesh Guptha, Dr P. Mahesh Kumar, Dr M. Sunil Kumar
}

Karpaga Vinayaga Institute of Dental Sciences, Madhuranthagam(Tk)

Chengalpattu (Dt) 603 308.Tamilnadu, India

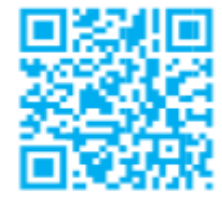

DOI: 10.37841/jidam_2021_V8_14_05

Address for Correspondence:

Dr P.K. Aravind, Postgraduate Student,

Karpaga Vinayaga Institute of Dental Sciences

Gst Road, Chinna Kolambakkam, Palayanoor (Po),

Madhuranthagam(Tk), Chengalpattu (Dt) 603308

Tamilnadu, India

Email id: pkaravindsha@gmail.com

Received: 19.11.2021 First Published: 12.12.2021

Accepted: 10.12.2021 Published: 27.12.2021

\begin{abstract}
The cornerstone of pediatric dental practice is successfully rehabilitating a preschooler with advanced carious lesions at esthetic level. Compromises such as decreased masticatory efficiency, impaired esthetics, and mispronunciation of labiodental sounds and development of atypical oral habits can occur due to the early loss of primary anterior teeth owing to trauma or caries. Parental desire is the most important element in deciding whether to place an anterior esthetic appliance to replace missing teeth. This case report details the fabrication of Groper's appliance for a 4 1/2-year-old child diagnosed with rampant caries.
\end{abstract}

KEYWORDS- Esthetic rehabilitation, Groper's appliance, Primary teeth, Space loss 
Aravind et al: Aesthetic Approach for extracted Upper Primary Incisors In A 4 1/2-Year-Old Child

\section{INTRODUCTION}

Rampant caries has been defined as a suddenly appearing, widespread, rapid burrowing type of caries, resulting in early involvement of the pulp and affecting those teeth usually regarded as immune to ordinary decay [Massler]. ${ }^{1}$ It is the most prevalent cause of lesions in the primary anterior teeth. This type of caries develops in very young infants due to prolonged or inappropriate eating practices. During sleep, the new-born can be breast fed or by a bottle containing a lot of fermentable carbohydrates. The sweet liquid substrate of the bottle collects around the maxillary incisors. Cariogenicity is strong in this sweet retentive environment. Caries is more likely to develop when salivary production is reduced while sleeping, teeth cleaning is neglected, and permitted nocturnal breastfeeding. The lesions begin on the labial surface of the anterior teeth and progress to posterior teeth. As a result of the diffuse demineralization, all primary anterior teeth are lost. ${ }^{2}$

Primary teeth are wrongly denoted as "temporary teeth" despite being very important for a child's overall health, mastication, aesthetics, pronunciation, personality, emotional well-being etc. Primary teeth have a major impact on one's quality of life. Early loss of primary anterior teeth impacts a child's growing dentition and presents a number of physiological and psychological problems. Early childhood caries and dental trauma are the major causes for loss of deciduous maxillary front tooth. ${ }^{3}$ With the exception of the mandibular primary incisor, rampant caries in the primary dentition is often connected to the tooth eruption sequence. The mandibular incisors are possibly more resistant to caries because they are in close contact with the secretions of the submandibular salivary glands and are cleansed by the tongue during bottle feeding. ${ }^{4}$

Prosthetic replacement of deciduous teeth is exceptionally important when a child has lost his/her deciduous teeth at a young age. To restore all functionality, including the child's aesthetics and mental advancement, the replacement should be designed in such a way the space maintainer does not interfere with the underlying successor teeth's eruption process. Removable or fixed functional space maintainers are the aesthetic solutions available for early loss of teeth. Fixed space maintainers are recommended because removable space maintainers are less desirable in young children due to issues with dental hygiene and relying on the child's compliance and cooperation to wear them. ${ }^{3}$ In recent years due to increased demands for aesthetics from parents, fixed functional space maintainers are the perfect solution for replacing deciduous anterior teeth that have prematurely exfoliated.

\section{CASE REPORT}

A male patient aged four and half years diagnosed with rampant caries reported with his parents to the Department of Pedodontics and Preventive Dentistry at Karpaga Vinayaga Institute of Dental Sciences, Madhurandhagam. With a chief complaint of a decayed tooth in the upper and lower front and back tooth region. History revealed that the patient had decayed teeth for six months, with no history of pain and sensitivity. The patient did not have any other oral habits.

The Patient's general examination revealed height as 95 centimetres, weight as 18 kilograms where the BMI score is 19.94 with a normal gait and straight posture. Intraoral examination (FIG $1 \mathrm{a}-\mathrm{c}$ ) of the patient revealed number of teeth as twenty with mesial step molar relation on both sides, dental caries in 54, 53, 52, 51, 61, 62, 63, 64, 71, 72, $73,74,75,81,82,83,84,85$.

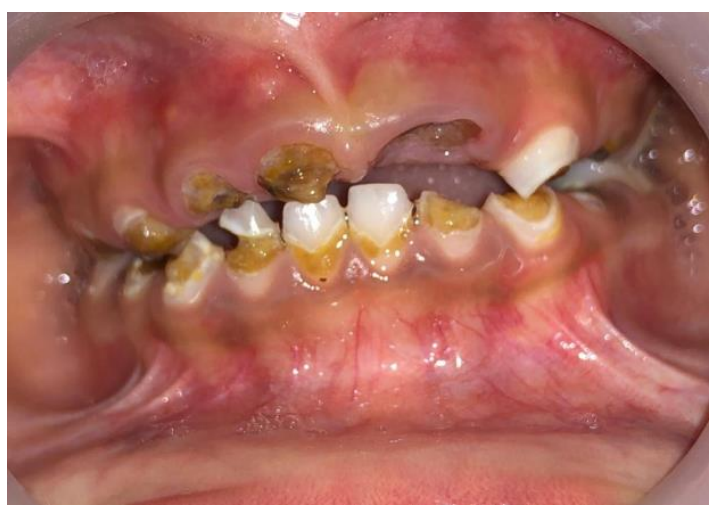

Fig 1a. Frontal view in occlusion

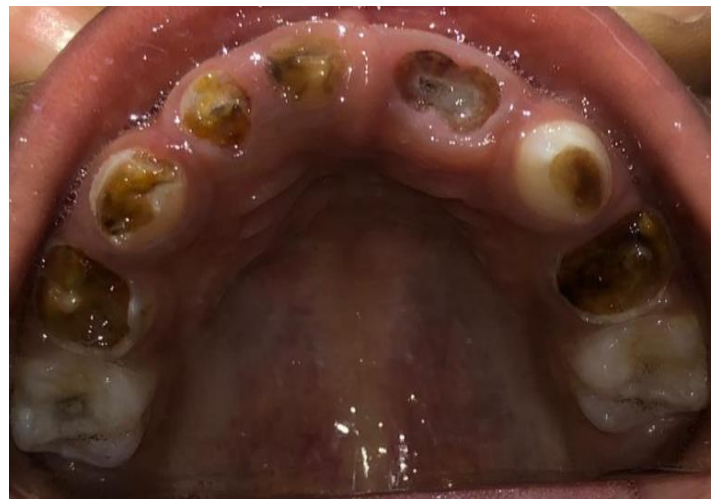

Fig 1b. Maxillary arch 


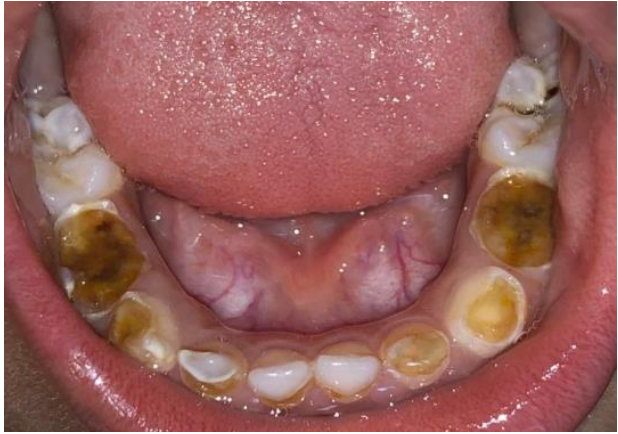

Fig 1c. Mandibular arch

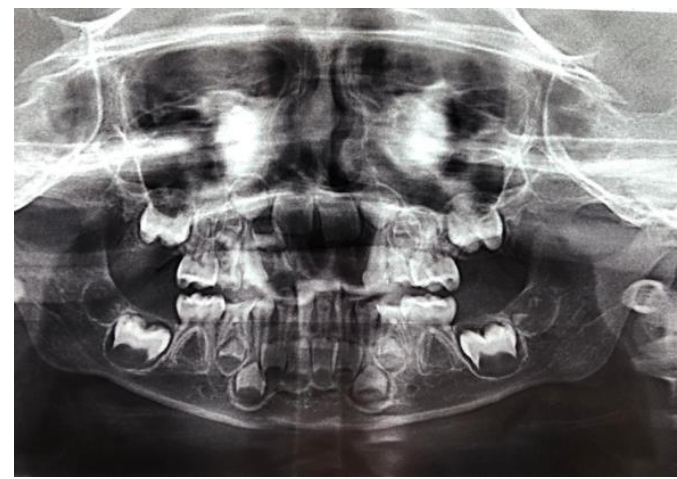

Fig 1d. Orthopantomogram

OPG (FIG 1d) revealed radiolucency involving enamel, dentin, and pulp in 52, 51, 61 and 62. Radiolucency approximating the pulp in $54,64,74,84,72,73,82,83$. There is evidence of fusion 61 and 62. Fusion (or syndontia) describes the joining of two (or more) independently developing primary or permanent teeth that began from their own unique tooth germs. Fusion leads to the formation of a single large tooth and reduces the total number of visible teeth in the affected arch by one (or more). Fusions can occur in both the primary and permanent dentitions. Fused teeth are at higher risk of developing dental caries along the line of crown fusion. ${ }^{5}$

Treatment plan has been explained to parent and informed consent has been obtained. Since the child was not responding for behaviour modification techniques. ${ }^{6}$ After taking opinion from Pediatrician \& Anesthetist treatment was planned under general anesthesia. Treatment procedures were started with pulpectomy in $54,64,72,73,74,82,83,84$ and then followed by Glass ionomer cement restoration (Type IX), Tooth preparation was done in 54, 64, 74, 84 and additionally preparation also done in 55 and 65 because it is selected as abutment for groper's appliance.

Stainless steel crowns were delivered in tooth number 54, 64, 74 and 84 . Additionally tooth preparation was done in
55 and 65 for groper's appliance because it is selected as abutment. Stainless crown in $55 \& 65$ placed in impression in their respective position and stabilized following which dental cast were made. A stainless-steel wire with a diameter of 0.036 " was utilized and adapted to the palate from 55 and 65 . The wire was positioned two millimetres beneath the gingival margin. Acrylic teeth were utilized in relation to 52,51,61, and 62 and attached to the stainless wire with the help of the cold cure. Patient came to the department after five days of alginate impression to receive the appliance. The gropers appliances insertion is done using cementation of stainless-steel crowns in 55,65 using Glass ionomer cement (Type I) (FIG 2a,b). The parents were satisfied with the appliance and were instructed to come for regular follow-up.

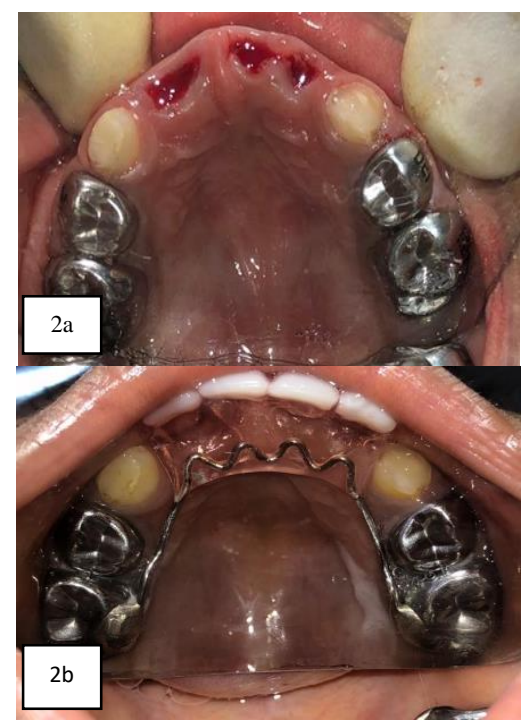

Fig 2a and $\mathrm{b}$. Before and after insertion of groper's appliance

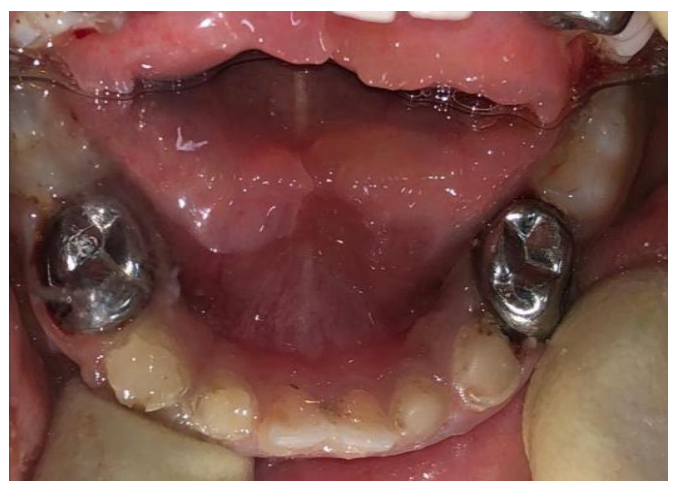

Fig 2c. Mandible occlusal view 


\section{DISCUSSION:}

Losing deciduous anterior teeth before the age of three might cause speech difficulties. So, in this case, scenario extracted maxillary incisors may results in loss of aesthetics. Missing primary anterior teeth should be replaced for several reasons; one of the most important reason is that it helps children's psychological development by restoring their appearance. ${ }^{3}$

The Groper appliance was developed in 1984 to minimize the aesthetic and functional consequences of early anterior tooth loss. The appliance is similar to a Nance holding arch, but instead of a palatal acrylic button in the rugae region, it uses acrylic plastic teeth coupled to a wire. A legitimate purpose to replace lost incisors is to restore a natural and pleasant look and so allow for appropriate psychological development. The primary drawback is that food debris accumulates. So, patients and their parents must be thoroughly educated on the value of dental hygiene. ${ }^{7}$

Aswanth et al. (2014) placed an anterior fixed functional space maintainer in a 4 1/2-year-old boy following extraction of severely decaying 52,51,61 and 62 teeth due to complaints of an unpleasant appearance, malnutrition, and altered speech. According to them, the aesthetic space maintainer is seamless and provides conformance when used by a young child patient ${ }^{8}$. In this case report patient achieved pleasant appearance because this space maintainer has numerous benefits, including improved aesthetics, improved masticatory, speaking efficiency and protection against the formation of undesirable mouth habits. The most significant drawback is the build-up of plaque and food particles $^{3}$. As a result, parents must be taught how to oversee their children's oral hygiene upkeep.

\section{CONCLUSION}

We discussed a treatment plan for early loss of primary anterior teeth. These appliances are nearly always viewed as extras, and where they go depends on the parent's preferences. The child's self-esteem soared when the gropers appliance restored his anterior aesthetics and function in only a few months. The patient and his parents were also given oral hygiene guidelines to practice at home.

\section{DECLARATION OF PATIENT CONSENT}

The authors certify that they have obtained all appropriate patient consent forms. In the form, the patient(s) has/have given his/her/their consent for his/her/their images and other clinical information to be reported in the journal. The patients understand that their names and initials will not be published and due efforts will be made to conceal their identity, but anonymity cannot be guaranteed.

\section{FINANCIAL SUPPORT AND SPONSORSHIP}

Nil

\section{CONFLICTS OF INTEREST}

There are no conflicts of interest.

\section{REFERNCES:}

1. Patel B. Evaluation of Knowledge, Attitude and Awareness of Pediatricians regarding Rampant Caries. Sch J Dent Sci. 2021 Jan;1:58-62.

2. Joybell CC, Ramesh K, Simon P, Mohan J, Ramesh M. Dental rehabilitation of a child with early childhood caries using Groper's appliance. J Pharm Bioallied Sci. 2015 Aug;7(Suppl 2):S704-7.

3. Anand S, Singh A, Jyoti D, Sulekha, Seal B. An esthetic approach for premature missing maxillary primary anterior teeth:A series of two case reports. Indian J Dent Sci 2021;13:205-8.

4. Yiu CK, Wei SH. Management of rampant caries in children. Quintessence Int. 1992 Mar;23(3):15968.

5. McDonald and Avery's. Acquired and Developmental Disturbances of the Teeth and Associated Oral Structures. In: Dean, J.A, editor. Dentistry for the Child and Adolescent. 11th ed. Elsevier Mosby. Missouri; 2021.p.42.

6. Raju VG (2019) A three-and-a-half-year-old patient with Prader-Willi syndrome: a case report on dental management with behavioural modification. Oral Health Care 4

7. Arikan V, Ocal D, Akcay M, Altug AT, Ozer L, Cesur E. Growth-enabling modified Groper appliance for the replacement of permanent maxillary incisors: Report of two cases. Australasian Orthodontic Journal. 2020 May;36(1):108-13.

8. Aswanth KP, Asokan S, Baby John J. Fixed functional space maintainer: A weight gainer: A case report. J Indian Acad Dent Spec 2014;1:25-7. 